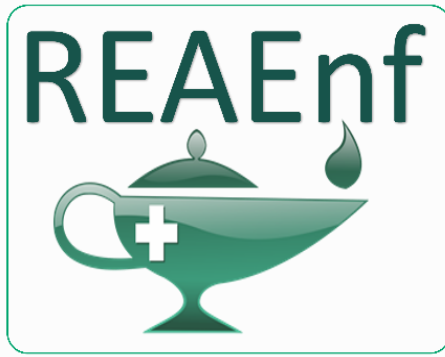

Revista Eletrônica Acervo Enfermagem
REVISÃO BIBLIOGRÁFICA

Recebido em: 1/2021

Aceito em: 2/2021

Publicado em: 3/2021

\title{
Sistemas de informação em saúde, o instrumento de apoio à gestão do SUS: aplicabilidade e desafios
}

\author{
Health information systems, the instrument to support SUS management: aplicability and \\ challenges
}

\section{Los sistemas de información en salud, un instrumento de apoyo a la gestión del SUS: aplicabilidad y desafíos}

Lucas lago Marques Saraiva1*, Francisco Alexandre Sena Ramos ${ }^{1}$, Gilvan Ferreira dos Santos ${ }^{1}$, Jabneela Vieira Pereira Vetorazo ${ }^{1}$.

Resumo: Esse artigo buscou abordar sobre os Sistemas de Informação em Saúde utilizado pelos profissionais na gestão de saúde no Sistema Único de Saúde. Observou-se que três são os pilares que formam um sistema de informação (SI): dado, informação e conhecimento. Para a saúde, uma informação contribui no processo decisório, uma vez que auxilia no conhecimento sobre as condições de saúde, morbimortalidade, fatores de riscos, condições demográficas e entre outras. O sistema atualmente utilizado em grande parte dos municípios é o e-SUS que é apoiado por dois sistemas de informação em saúde para Atenção Básica (SISAB), o sistema de informação nacional e o sistema e-SUS na Atenção Básica. Essas informações colhidas pelos sistemas são imprescindíveis ao planejamento, à implantação, à implementação e à avaliação de ações e serviços de saúde, estas são capazes de identificar problemas individuais e coletivos. Muitas vezes, os gestores e profissionais de saúde não utilizam os sistemas de informação de maneira correta, sendo muitas vezes incipiente para o processo decisório da gestão. Esses profissionais usam e compartilham informações e dados, no entanto, enfrentam desafios no uso dessa tecnologia como substrato para a produção do conhecimento e decisão.

Palavras-chave: Sistemas de informação, Gestão em saúde, Enfermagem.

\begin{abstract}
This article sought to address the Health Information Systems used by professionals in health management in the Unified Health System. It was observed that three are the pillars that form an information system (IS): data, information and knowledge. For health, information contributes to the decision-making process, since it helps in the knowledge about health conditions, morbidity and mortality, risk factors, demographic conditions and among others. The system currently used in most municipalities is the e-SUS, which is supported by two health information systems for primary care (SISAB), the national information system and the e-SUS system in primary care. This information collected by the systems is essential to the planning, implementation, implementation and evaluation of health actions and services, they are able to identify individual and collective problems. Often, managers and health professionals do not use information systems correctly, and are often incipient in the management decision-making process. These professionals use and share information and data, however, they face challenges in using this technology as a substrate for the production of knowledge and decision.
\end{abstract}

Key words: Information systems, Health management, Nursing.

${ }^{1}$ Centro Universitário Aparício Carvalho (FIMCA), Porto Velho - RO.

*E-mail: alexandre.gilvan.lucas@gmail.com 
Resumen: Este artículo buscó abordar los Sistemas de Información en Salud que utilizan los profesionales de la gestión en salud en el Sistema Único de Salud, se observó que tres son los pilares que conforman un sistema de información (SI): datos, información y conocimiento. Para la salud, la información contribuye a la toma de decisiones, ya que ayuda en el conocimiento sobre las condiciones de salud, morbilidad y mortalidad, factores de riesgo, condiciones demográficas y otros. El sistema que se utiliza actualmente en la mayoría de los municipios es el e-SUS, que se apoya en dos sistemas de información en salud para la atención primaria (SISAB), el sistema de información nacional y el sistema e-SUS en la atención primaria. Esta información recolectada por los sistemas es fundamental para planificar, implementar, implementar y evaluar acciones y servicios de salud, son capaces de identificar problemas individuales y colectivos. A menudo, los gerentes y profesionales de la salud no utilizan correctamente los sistemas de información y, a menudo, son incipientes en el proceso de toma de decisiones de gestión. Estos profesionales usan y comparten información y datos, sin embargo, enfrentan desafíos en el uso de esta tecnología como sustrato para la producción de conocimiento y decisión.

Palabras clave: Sistemas de información, Gestión sanitaria, Enfermería.

\section{INTRODUÇÃO}

O Sistema Único de Saúde (SUS) compreende à saúde como direito do cidadão e dever do Estado, através de uma rede de ações e serviços regionalizados e hierarquizados (BRASIL, 2009). Para que isso ocorra, o SUS sugere o uso do conceito ampliado de saúde, que associa as condições de vida, orienta a formulação e a implementação de estratégias que viabilizem um serviço de saúde universal, integral, eficiente, com equidade e participação popular (JUNIOR SCSG e ALMEIDA RT, 2009).

Para que haja uma formulação de políticas de saúde, a avaliação e o monitoramento das condições de saúde da população são peças chaves para que haja uma visualização global do sucesso dos programas elaborados para a população. Embora tais indicadores sejam úteis em contextos determinados de problemas-decisões, ter um conjunto de índices que classifiquem as diferentes dimensões do processo saúde-doença possibilita gerar a concepção de um cenário completo e instigante da saúde (SIQUEIRA MC, 2015).

Sabendo que inúmeros fatores podem influenciar o processo de saúde-doença e que a demanda de usuários tem aumentado, existe a necessidade de que se utilizem os indicadores de assistência à saúde, para que se possa estimar o desempenho do sistema de saúde em conduzir as políticas públicas, bem como sua tomada de decisões. Deste modo, a inclusão das tecnologias de informação nos processos de saúde tem sido de fundamental relevância (SANTOS SR, et al., 2014).

Os Sistemas de Informação da Saúde (SIS) são uma estrutura que garante a obtenção e a transformação de dados em informação, no qual acontece o processo de seleção, coleta, classificação, armazenamento, análise, divulgação e recuperação de dados de saúde (FRANCO JLV, 2015).

O interesse em abordar o tema surgiu a partir da observação, em campo de estágio, de um grande quantitativo de profissionais que tinham dificuldade em repassar as informações de forma precisa, bem como manusear os programas de informatizações nas redes públicas, em hospitais e unidades básicas de saúde. Sendo perceptível o grande número de reclamações de profissionais a respeito do sistema de informação, o presente estudo teve por objetivo principal abordar sobre o SIS utilizado pelos profissionais de saúde na gestão de saúde no Sistema Único de Saúde.

\section{REVISÃO BIBLIOGRÁFICA}

Na década de 1950, houve o surgimento dos sistemas de informação no Oriente, Continente Europeu e Continente Norte americano, em que possibilitavam realizar a coleta, armazenamento e sistematização de dados institucionais, de forma a fornecer suporte as decisões. No entanto, estes eram limitados ao nível gerencial e administrativo, somente dez anos mais tarde foram aceitas as possibilidades de automatizar as ações de saúde e com isso novos sistemas foram criados e adaptados para o uso de informações hospitalares (CAVALCANTE RB, et al., 2011). 
No Brasil, sua utilização se deu tardiamente, sendo primeiramente utilizados modelos empresariais delimitados a atividades administrativas do hospital. Somente na década de 90 que os sistemas de informação foram voltados aos pacientes, atualmente estes são direcionados ao suporte do cuidado de qualidade e promover informações fidedignas para que possam fundamentar decisões dos gestores e dos profissionais da assistência (TARGINO MG, 2009).

A informação se tornou um importante instrumento para tomada de decisões na assistência a saúde, pois representa um fator desencadeador do processo "informação-decisão-ação", na qual sintetiza a dinâmica de suas atividades que se iniciam através da informação de um indício ou suspeita de um caso de doença ou algum agravo (BRASIL, 2005).

De acordo com Pinheiro AL, et al. (2016), os bancos de dados e os sistemas de informações em saúde são ferramentas fundamentais para o planejamento e avaliação das políticas de saúde, bem como dos serviços, redes e sistemas de saúde. A gestão do Sistema Único de Saúde (SUS) é o processo que tange as tomadas de decisões na implementação da política de saúde, além do desenvolvimento de toda a logística e da tecnologia de regulação baseada nas necessidades prioritárias, vulnerabilidades e riscos, visando ainda a necessidade de mediação política e definição de diretrizes e critérios para participação e controle social (CARVALHO ALB, et al., 2012).

Três são os pilares que formam um sistema de informação (SI): dado, informação e conhecimento. $O$ dado pode ser considerado como o elemento mais simples do processo; a informação é composta por dados com significados para quem os vê; os conhecimentos adquiridos após convenções experiências e percepção cognitiva transformam em conhecimento uma dada realidade (SIQUEIRA MC, 2015).

$\mathrm{Na}$ década de 90 , esses sistemas surgiram para transformar as informações e dados para a forma eletrônica, podendo assim ocorrer uma redução de custos e diminuir a dependência de papéis. Além de facilitar o atendimento devido às informações serem mais fáceis de armazenar, copiar, manipular e com disponível nosistema que estiver (CAVALCANTE RB, et al., 2011).

O processo decisório depende de informações para seu prosseguimento, uma vez que esse auxilia no conhecimento sobre as condições de saúde, morbimortalidade, fatores de riscos, condições demográficas e entre outras. Para que isso ocorra, esses sistemas utilizam de todo um aparato tecnológico como o hardware, software, banco de dados, redes, procedimentos e pessoas, de forma interligada e dependente um do outro para seu pleno funcionamento para o processo de trabalho (NETTO JJM, et al., 2016).

O SIS deve disponibilizar um suporte necessário para o planejamento, processo decisório e ações dos gestores, para que não se baseie em dados subjetivos, conhecimentos ultrapassados e hipóteses (BRASIL, 2005). Por ser parte do sistema de saúde, o SIS, constituído por vários subsistemas em que busca facilitar a formulação e avaliação das políticas, planos e programas de saúde, no qual garante obter e transformar dados em informação de forma sucinta, em que os profissionais envolvidos conseguem esse resultado através de todo 0 processo de seleção, coleta, classificação, armazenamento, análise, divulgação e recuperação desses dados (SANTIAGO AX, et al., 2008).

De forma sucinta, um dos principais objetivos do SIS é, na concepção do Sistema Único de Saúde, é viabilizar a análise da situação de saúde no nível local, considerando as condições de vida da população na determinação de saúde-doença (BRASIL, 2005). Conforme Siqueira MC (2015), quando existe esse envolvimento dos profissionais na construção dos instrumentos de coleta, captação de dados de forma correta e processamento da informação são atitudes que atestam o domínio do profissional na área de conhecimento.

O SIS foi criado para auxiliar na melhoria da qualidade e da produtividade da assistência a saúde, em que possa viabilizar a realização de pesquisas e atividades de ensino. Sua utilização fornece aos profissionais de saúde um desempenho eficaz, em que integram a informação, facilitando a comunicação, coordenando ações e fornecendo apoio aos demais setores de saúde (CARVALHO JA, 2010). Para o autor, a estratégia de gestão é fundamental para que haja sucesso na instituição, em que requer o saber operacional e eficácia em seu uso. 
Na saúde são utilizados diversos SIS pelas organizações de saúde, seja ela de esfera pública e privada, implantadas com o intuito de reduzir custos e efetivar a qualidade dos serviços ofertados a população. Os principais sistemas de informação em saúde utilizados são o Sistema do Cadastro Nacional de Estabelecimentos de Saúde (SCNES), Sistema de Informações Ambulatoriais (SAI/SUS), Sistema de Informações Hospitalares (SIH/SUS), Sistema Nacional de Agravo de Notificação (SINAN), Sistema de Informações sobre Nascidos Vivos (SINASC) e Sistema de Informação sobre Mortalidade (SIM) (GARCIA PT e REIS RS, 2016).

O Sistema do Cadastro Nacional de Estabelecimentos de Saúde cadastra todos os estabelecimentos de saúde da rede pública e privada, para assim manter atualizados os bancos de dados locais e federais. O Sistema de Informações Ambulatoriais processa todos os dados ambulatoriais da Atenção Básica, Média e Alta Complexidade, essas informações vão para o banco de dados nacional dos atendimentos ambulatoriais onde direcionam o processo de planejamento, programação, regulação, avaliação e controle desses serviços de saúde (BRASIL, 2009; ANDRADE AG, 2011).

O Sistema de Informações Hospitalares registra os atendimentos de internações que foram financiadas pelo SUS, ainda dispõe informações de cada hospital da rede do SUS como principais causas de internações, procedimentos mais frequentes, quantia de leitos dentre outras. O Sistema Nacional de Agravo de Notificação coleta, transmite e dissemina dados gerados pela vigilância epidemiológica em todas as esferas do governo (PEREIRA MN, et al., 2013; BRASIL, 2006).

O Sistema de Informações sobre Nascidos Vivos agrupa dados epidemiológicos referentes aos nascidos em todo território nacional, além de disponibilizar informações de morbidade, mortalidade tanto infantil como materna, situações de atenção ao parto dentre outras. O Sistema de Informação sobre Mortalidade dispõe de dados a partir de 1979, sendo o mais antigo sistema. Com essas informações é possível ocorrer análises das situações das regiões, planejamento e avaliações de ações e programas nas determinadas áreas (BRASIL, 2004).

Esses sistemas prestam apoio a parte administrativa e clínica das consultas, participam desde a coleta de dados, armazena todas as informações, processa essas informações e auxilia no diagnóstico, estão presentes também na parte de prescrição de medicações e diversas outras funções, estando assim em diversos setores e todas as informações ficam organizadas nos prontuários eletrônicos dos pacientes (CAVALCANTE RB, et al., 2011).

Existe um expressivo número de variáveis internas e externas que afetam os processos de saúdedoença e na organização/administração dos programas, serviços e unidades de saúde. Com isso, existem obstáculos para a padronização de insumos, métodos, técnicas e áreas afins devido a diversidade de categorias profissionais (GAVA M, et al., 2016).

Para seu bom funcionamento seria necessário a boa gestão dos sistemas e serviços de saúde, sendo definido como compartilhamento de uma única base de dados e que envolvesse todos os níveis de hierarquia organizacional de saúde (RIBEIRO NT, et al., 2016).

Ainda que possua todas essas funções e utilidade, existe uma grande resistência por parte de alguns profissionais ao aderir essa ferramenta. O desconhecimento da tecnologia, a deficiência no ensino da informática nas escolas e tardiamente nas faculdades, são alguns dos desafios que precisam ser vencidos para que ocorra a implantação de um sistema eletrônico de saúde efetivo e funcional (SILVA SF, 2011).

Outros desafios encontrados são a segurança dos dados e a falta de informações pelos profissionais sobre o sistema, em que o acesso as informações eletrônicas devem ser restritas e obedecer às normas para registro da assistência ao paciente, para que cada profissional só tenha acesso às informações referentes ao seu atendimento (CAVALCANTE J, 2015).

Em relação à desinformação por parte dos profissionais, os programas muitas vezes não correspondem a real necessidade do profissional, ou por ele não encontrar as funções e opções necessárias, ou por quem criou o sistema não ser relacionado à área da saúde e ter criado ferramentas inaplicáveis (CAVALCANTE RB, et al., 2011).

REAEnf/EJNC | Vol. 9 | e6418 | DOI: https://doi.org/10.25248/REAenf.e6418.2021 Página 4 de 6 
O Departamento de Informática do Sistema Único de Saúde (DATASUS) foi criado em 1991, juntamente com a criação da Fundação Nacional de Saúde (FUNASA), elaborada pelo Decreto de oㅜ 100 de 16 de abril de 1991. Conforme o DATASUS (2020), essa fundação começou a controlar e processar as contas do sistema de saúde em que era gerenciada anteriormente pela Empresa de Tecnologia e Informações da Previdência Social. A partir de então, é formalizada pelo DATASUS, sua criação e competências, em que tem como responsabilidade munir os órgãos do SUS de sistemas de informação e suporte de informática, necessários ao processo de planejamento, operação e controle (DATASUS, 2020).

O sistema atualmente utilizado em grande parte dos municípios é o e-SUS, este que é apoiado por dois sistemas de informação em saúde para Atenção Básica (SISAB), o sistema de informação nacional e o sistema e-SUS Atenção Básica, composto por sistemas de software que instrumentalizam o processo de trabalho nas unidades básicas de saúde (UBS) (MINISTÉRIO DA SAÚDE, 2015).

Tal sistema foi elaborado para melhorar e substituir o uso das informações do SIAB para gestores, profissionais e cidadãos, no qual o Departamento de Atenção Básica (DAB) que adotou essa estratégia utilizam os sistemas de captação de dados, Coleta de Dados Simplificada (CDS-AB) e Prontuário Eletrônico do Cidadão (PEC), que alimentam o Sistema de Informação em Saúde da Atenção Básica (SISAB) (BRASIL, 2014).

Devido às várias subdivisões da área da saúde e suas diferentes necessidades, ocorre um exagero de softwares e planilhas que não tem uma visão sistêmica, portanto não são interligados. Essa não integração dos sistemas leva as informações a se tornarem menos confiáveis, devido à necessidade de coleta de dados ser em bases diferentes e esses mesmo dados serem processados em outro ambiente, esse fracionamento de informações cria um risco grande de erros ou perda de dados (BITTAR OJN, 2018).

O sistema e-SUS registram as informações em saúde a caráter individual do cidadão, em que permite o acompanhamento do usuário em cada aceso à rede de atendimento. Este permite a interface com outros sistemas utilizados no SUS, evitando assim que refaça a alimentação dos dados comuns nas fichas/sistemas (BRASIL, 2014).

Essas informações colhidas pelos sistemas são imprescindíveis ao planejamento, à implantação, à implementação e à avaliação de ações e serviços de saúde, estas são capazes de identificar problemas individuais e coletivos. O principal objetivo dos SIS é dar apoio e provocar mudanças de forma que melhore o funcionamento dos processos de trabalho e o cuidado a saúde (TARGINO MG, 2009).

A principal ferramenta do SIS utilizada pelos médicos e equipes de saúde é o prontuário eletrônico do paciente (PEP), em que estes profissionais precisam lidar com esse sistema nas suas atividades diárias. Apesar dos inúmeros benefícios esse avanço exige além do investimento de alto custo, capacitações profissionais com intuito de melhorar as práticas de registro nessas ferramentas digitais (BITTAR OJN, 2018).

Ainda que haja grandes vantagens no uso dos sistemas e haja disponibilidade de computadores e internet, muitos municípios preferem continuar na forma tradicional de comunicação, como correio ou telefone. $O$ aperfeiçoamento e qualificação desses profissionais é muito importante para que entendam que conhecer o funcionamento do SIS facilita o processo de trabalho e transmissão da informação, bem como minimiza os erros e a demora pelo método tradicional. Outras limitações que podem ser identificadas são a falta de materiais e equipamentos, as constantes falhas na conectividade que dificultam o processo de trabalho e exportação desses dados, e a dificuldade de acesso à internet que compromete todo o processo para qual o sistema de informação foi elaborado (MACHADO CS e CATTAFESTA M, 2019).

Contudo, o emprego das tecnologias pode ser otimizado com a implementação de políticas que busquem a inovação da informação em que acelerem o processo de inclusão de tecnologias da informação e comunicação, e que complementam e adaptem o apoio apropriado ao processo decisório, à conscientização quanto a sua importância e ao envolvimento dos profissionais e gestores de saúde (MACHADO CS e CATTAFESTA M, 2019). 


\section{CONSIDERAÇÕES FINAIS}

Os gestores de saúde em suas diversas funções e diante da complexidade da área, têm se confrontado no seu cotidiano com diversos desafios, dentre eles o uso das informações como recursos primordiais para o desenvolvimento do conhecimento para a tomada de decisão. Muitas vezes, os gestores e profissionais de saúde não utilizam os sistemas de informação de maneira correta, sendo muitas vezes incipiente para o processo decisório da gestão. Contudo, verifica-se que o SIS tem proporcionado muitos pontos benéficos para o setor da saúde, mas também algumas implicações. É imprescindível o investimento em capacitações específicas nas instituições de saúde, realizando assim a aproximação do profissional com o sistema de informação.

\section{REFERÊNCIAS}

1. ANDRADE AG. O Sistema de Informações Ambulatoriais como Instrumento para a Regionalização Em Saúde. 2011. Monografia (Especialização em Saúde Pública) - Departamento de Saúde Coletiva, Centro de Pesquisas Aggey Magalhães, Fundação Oswaldo Cruz, Recife, 2011.

2. BITTAR OJ, et al. Sistemas de Informação em Saúde e sua Complexidade. Revista Administração em Saúde, 2018, 18(70).

3. BRASIL. Ministério da Saúde. Biblioteca Virtual em Saúde. Diretriz Nacional de Implantação da Estratégia e-SUS Atenção Básica. Brasília, 2014

4. BRASIL. Ministério da Saúde. Secretária de Vigilância em Saúde. Departamento de Vigilância Epidemiológica. Sistema de Informação de Agravos de Notificação. Série A. Normas e Manuais Técnicos. 1a ed. Brasília, 2006.

5. BRASIL. Ministério da Saúde. Secretaria de Vigilância em Saúde. Guia de Vigilância Epidemiológica. $2^{a}$ ed. Brasília, 2005.

6. BRASIL. Ministério da Saúde. Secretaria Executiva. Departamento de Apoio à Descentralização. O SUS no seu Município garantindo saúde para todos. $2^{\underline{a}}$ ed. Brasília, 2009.

7. BRASIL. Ministério da Saúde. Sistemas de Informações sobre Mortalidade (SIM) de Nascidos Vivos (SINASC). Brasília, 2004.

8. CARVALHO ALB, et al. A gestão do SUS e as práticas de monitoramento e avaliação: possibilidades e desafios para a construção de uma agenda estratégica. Ciência \& Saúde Coletiva, 2012,17(4): 901-911.

9. CARVALHO JA. Tecnologias e sistemas de informação: uma área científica orientada às necessidades de conhecimento dos profissionais envolvidos na contínua transformação das organizações através das tecnologias da informação. Revista Eletrônica Científica de Informação, 2010.

10. CAVALCANTE J. Tecnologia e Sistemas de Informação. São Francisco, 2015.

11. CAVALCANTE RB, et al. Sistemas de Informação em Saúde: Possibilidades e Desafios. Revista de Enfermagem da UFSM, 2011, 1(2):290-299.

12. DATASUS. Ministério da Saúde. Sobre o DATASUS. Brasil, 2020.

13. FRANCO JLF. Sistemas de Informação. São Paulo, 2015.

14. GARCIA PT, REIS RS. Gestão Pública em Saúde: Sistemas de Informação de Apoio à Gestão em Saúde. 1ª ed. São Luís: EDUFMA, 2016.

15. GAVA M, et al. Incorporação da tecnologia na Atenção Básica do SUS no Nordeste do Brasil: expectativas e experiências. Cien Saude Colet., 2016; 21(3):891-902.

16. JUNIOR SCSG, ALMEIDA RT. Modelo de simulação para estimar a infraestrutura necessária à assistência oncológica no sistema público de saúde. Revista Panamericana Salude Publica, 2009, 25(2): 113-119.

17. MINISTÉRIODA SAÚDE. Sistema e-SUS Atenção Básica. Manual de exportação. Brasília, 2015.

18. MACHADO CS, CATTAFESTA M. Benefícios, dificuldades e desafios dos sistemas de informações para a gestão no Sistema Único de Saúde. Revista Brasileira de Pesquisa em Saúde, 2019, 21(1): 124-134.

19. NETTO JJM, et al. Uso de instrumentos enquanto tecnologia para a saúde. Saúde em Redes, 2016, 2(1): 65-72.

20. PEREIRA MN, et al. Sistema de Informações Hospitalares do Sistema Único de Saúde (SIH-SUS): uma avaliação do seu desempenho para a identificação do near miss materno. Cadernos de Saúde Pública, 2013, 29(7): 13331345.

21. PINHEIRO ALS, et al. Gestão da Saúde: O uso dos sistemas de informação e o compartilhamento de conhecimento para a toma de decisão. Revista Texto \& Contexto Enfermagem, 2016, 5(3): e3440015.

22. RIBEIRO NT, et al. A análise de adesão de competências organizacionais relacionadas à pós-implementação de sistemas ERP em hospitais públicos da administração direta de São Paulo - SP. Espacio, 2016, 37(19):3.

23. SANTIAGO AX, et al. Evolução histórica dos principais indicadores de saúde do Município do Sobral, Ceará, Brasil: 1998 a 2007. Revista de Políticas Públicas SANARE, 7(1): 12-19.

24. SANTOS SR, et al. Sistema de Informação em Saúde: Gestão e assistência no Sistema Único de Saúde. Revista Cogitare Enfermagem, 2014, 19(4): 833-840.

25. SILVA SF. Organização de redes regionalizadas e integradas de atenção à saúde: desafios do Sistema Único de Saúde (Brasil). Revista Ciência \& Saúde coletiva, 2011, 16(6): 2753-2762.

26. SIQUEIRA MC. Gestão estratégica da informação. Rio de Janeiro: Brasport, 2015.

27. TARGINO MG. Informações em Saúde: Potencialidades e Limitações. Revista Informação \& Informação, 2009, 14(1):52-81. 